\title{
Anaemia in Quick Diagnostic Unit
}

\author{
Charlotte Stenqvist* and Thomas Andersen Schmidt \\ The Emergency Department, Holbaek University Hospital, Denmark
}

Received: July 25, 2014; Accepted: September 21, 2014; Published: October 12, 2014

*Corresponding author: Charlotte Stenqvist, The Emergency Department, Holbaek University Hospital, Smedelundsgade 60, 4300 Holbaek, Denmark, Tel: +45 283177 86; E-mail: charlotte_stenqvist@hotmail.com

\begin{abstract}
Introduction: In a traditional in-hospital setting not least patients with chemotherapy induced anaemia experience a time burden when in need of a red blood cell transfusion (RBCT). The establishment of a Quick Diagnostic Unit (QDU) in an Emergency Department (ED) setting can allow expeditious blood transfusion of anaemic patients. The purpose of the study was to establish the mode of referral, describe the underlying diseases and the haemoglobin $(\mathrm{Hb})$ level of the referred patients.
\end{abstract}

Methods: Chart review of an 8-month period.

Results: We included 148 admissions. Twenty-five patients were admitted more than once, representing 108 individual patients. In the given period we treated around 4 patients each week. Three-fourths of the patients only stayed for a few hours.

Seventy one per cent of the patients were referred to hospital by their general practitioner and $18 \%$ of the patients came from oncological departments.

Fifty-five patients had a diagnosed cancer. Fifty-three patients had a non-oncological disease. The mean age for oncological patients was (mean \pm SEM) $73.8 \pm 1.3(n=55)$ years and for non-oncological patients $75 \pm 1.8$ years $(n=53)(p>0.6)$. Oncological patients were given RBCT at a $\mathrm{Hb}$ level of $5.0 \pm 0.09 \mathrm{mMol} / \mathrm{L}$. Non-oncological patients received RBCT at a $\mathrm{Hb}$ level of $4.7 \pm 0.07 \mathrm{mMol} / \mathrm{L}(\mathrm{p}<0.05)$. On average patients with malignant disease tended to receive less blood than patients with non-oncological diseases $(p=0.06)$, i.e. $2.2 \pm$ 0.1 vs. $2.5 \pm 0.1$ portions of RBCT per contact.

Conclusion: Non-trauma related RBCT may be given expeditiously in a QDU integrated in an ED setting to elderly patients. On average oncological patients received RBCT at a higher Hb level than other anaemic patients. The transfusion trigger for patients with non-oncological disease appears to comply with national guidelines.

Keywords: Red Blood Cell Transfusion; Cancer-induced Anaemia; Anaemia; Quick Diagnostic Unit

\section{Introduction}

In 2012 a Quick Diagnostic Unit (QDU) integrated in an Emergency Department (ED) setting was established at Holbaek University Hospital to allow expeditious treatment of less demanding admittances and to generally relieve the General Internal Medicine ward. The QDU is integrated both organizationally and physically in the ED. The scope of the
QDU is to receive patients referred by general practitioners or specialized departments at other hospitals via the ED for suspected severe disease in a stable condition, or minor medical ailments (deep venous thrombosis, anaemia etc.) that cannot be handled in an outpatient setting, but do not require a traditional admission. The QDU is also a buffer for ED overcrowding [1].

The establishment of a QDU in an ED setting has thus, allowed expeditious blood transfusion of anaemic patients. Before the establishment of the QDU in the ED all patients were transfused at the General Internal Medicine ward, because there are no outpatients infusion centers in our region of Denmark. That required a full lengthy, hospital admission with a clean bed for ambulatory patients and a lot of paperwork. The QDU transfusion model is a more expeditious, less bureaucratic alternative to standard care.

The National Board of Health in Denmark recommends blood transfusion when the haemoglobin ( $\mathrm{Hb}$ ) level is below 4.5 $\mathrm{mMol} / \mathrm{L}$, and no transfusion over $6.0 \mathrm{mMol} / \mathrm{L}$. Between a Hb level of 4.5-6.0 mMol/L transfusions can be performed on patients with malignant disease or patients with severe ischemic heart disease. Upon admission of a patient, a QDU physician confirms or rejects the indication for blood transfusion based upon point of care $\mathrm{Hb}$ evaluation.

Patients are referred to the QDU via the ED proper, and in the case of blood transfusion they are usually given a scheduled time to come the following day. Contrary to the ED setting, transfusions in the current context are never trauma related. There is no waiting time apart from the compatibility procedure. To minimize institutionalization, transfusion patients remain in their own clothing.

If the cause of anaemia was uncertain some patients returned to their general practitioner for further investigation, while others were referred to other departments in an outpatient setting for further investigation. Patients having an uncertain cause of anaemia can choose for themselves whether they want further investigation. If the medical history was suspicious of abdominal bleeding/cancer, patients were referred to the Department of Surgery for endoscopy. If blood tests indicated anaemia unrelated to bleeding, appropriate tests was performed.

In the US, it is proved that patients with chemotherapy 
induced anaemia experience an important time burden when being treated with red blood cell transfusion (RBCT) in addition to the time burden already added by chemotherapy [2]. It is likely that the current way of expeditious transfusions may increase these patients' quality of life because they can avoid longer hospitalization.

The purpose of the study was to establish the mode of referral for blood transfusion patients and to describe the underlying diseases and the $\mathrm{Hb}$ level of the referred patients.

\section{Methods}

Holbaek University Hospital is a 300 bed regional hospital with a catchment area of approximately 200,000 persons. The QDU integrated in the ED setting accommodates 16 patients and is comprised of 10 beds and six ambulatory seats/chairs. It is manned by physicians who are all ED employees, i.e. a Chief Physician, an additional rotating senior physician and an intern during daytime 08:00-16:00 (8 am - $4 \mathrm{pm})$, three nurses and a secretary. After 16:00 (4 pm) to the following morning, the QDU is manned by the physicians on call in the ED proper. During daytime shifts the staff rotates between working in the ED and the QDU.

In the QDU integrated in the ED setting 500 patients are admitted and discharged per month with a turnover of $\sim 1$ patient per accommodation (bed or seat/chair) per day [3]. The QDU practically sees all non-bleeding patients referred because of non-trauma-related anaemia. Yearly this amounted to 220 patients.

The QDU makes use of the ED's own Point of Care laboratory (POCT) manned by two bio analysts from 08:00-22:00 (8 am-10 pm), as well as an x-ray facility manned from 10:00-18:00 (10 am-6 pm). Additionally the Department of Radiology provides the QDU with more advanced diagnostic procedures such as e.g. CAT scans or MRI scans on a fast track basis. There is easy access to additional specialist evaluations from the ED staff or, albeit rarely, from various in house specialists.

The current study is a chart review of an 8-month period (Apr-Nov 2012). The study underwent Institutional Review Board approval. Adult patients ( $>18$ years old) with the diagnosis of anaemia or the procedure code for blood transfusion were included. All patients had a known diagnosis of non-traumarelated anaemia without active bleeding and presented with symptoms of fatigue, dyspnoea and palpitations. We observed the included patients' gender, age, underlying disease, transfusion threshold, mode of referral and their visiting frequency.

\section{Statistics}

Values were given as mean \pm SEM. Significance was evaluated using Student's two-tailed t-test for unpaired observations. The level of significance was ascertained at $\mathrm{p}<0.05$.

\section{Results}

Among 159 admissions, ten were excluded due to lack of an indication for RBCT, and one admitted patient was mistakenly given the procedure code for blood transfusion. He had a deep venous thrombosis. We included 148 admissions, amounting to 108 individual patients. Twenty-five patients $(15.7 \%)$ were admitted more than once. The excluded patients had a Hb level from 5.3 to $7.4 \mathrm{mMol} / \mathrm{L}$, with a mean $\mathrm{Hb}$ level of $6.2 \mathrm{mMol} / \mathrm{L}$ and did not present an indication for red blood cell transfusion as established by the transfusion requirements of the National Board of Health in Denmark.

All of these encounters were managed in the QDU. Although brief the encounters nevertheless formally count as admissions for registration purposes.

In a period of 8 months in 2011 prior to the establishment of QDU there were 163 admissions to Holbaek Hospital due to transfusion requiring anaemia. The total number of patients with transfusion requiring anaemia admitted to Holbaek Hospital including the QDU during the 8-month period in 2012 was 191 patients. The QDU transfused 148 patients, relieving the General Internal Medicine ward of $77 \%$ of the admissions related to transfusion requiring anaemia $[4,5]$. There were only 43 admissions to the General Internal Medicine ward during this period of time with transfusion requiring anaemia.

Seventy one percent of the patients were referred to hospital by their general practitioner and $18 \%$ of the patients were referred from oncological departments. Cardiology departments and hematological departments referred $2.7 \%$ and $3.3 \%$ of the patients. Of all referrals only $3.3 \%$ were unclassified.

In the given period we treated around 4 patients each week. Length of stay amounted to 2-4 hours per transfusion and depended on the number of transfusions needed. If more than 3 red blood cell transfusions (RBCT) was indicated, the patient returned the following day for RBCT completion. For this period of time a fourth of the patients returned the following day. Threefourths of the patients were discharged the same day.

Fifty-five patients had a diagnosed cancer, 29 were men and 26 were women. Fifty-three patients had a non-oncological disease, 26 were men and 27 were women (Table 1). The mean age for oncological patients was $73.8 \pm 1.3(n=55)$ years and for non-oncological patients $75 \pm 1.8$ years $(n=53)(p>0.6)$.

Oncological patients were given RBCT at a mean $\mathrm{Hb}$ level of $5.0 \pm 0.09 \mathrm{mMol} / \mathrm{L}$. Non-oncological patients received RBCT at a mean $\mathrm{Hb}$ level of $4.7 \pm 0.07 \mathrm{mMol} / \mathrm{L}(\mathrm{p}<0.05)$.

On average, patients with malignant disease tended to receive less blood than patients with non-oncological diseases, i.e. $2.2 \pm 0.1$ vs. $2.5 \pm 0.1$ portions of RBCT per contact $(p=0.06)$. This however in clinical practice amounts to an average of two portions of RBCT for both patient categories.

Twenty-five patients were admitted more than once, on average they came every 42nd day. Sixteen of them were men, and 9 were women. Patients receiving multiple transfusions had abdominal and rheumatologic disease to a greater extent than those receiving a single transfusion (Figure 1). 
Table 1: Disease characteristics of the included patients in decreasing order.

Abdominal disease included ulcer, diverticula and esophageal varices. Rheumatological diseases requiring transfusion were Wegener's granulomatosis, Rheumatoid arthritis and anaemia caused by treatment with Methotrexate.

\begin{tabular}{|l|c|c|}
\hline Disease & $\begin{array}{c}\text { Number of } \\
\text { patients }\end{array}$ & $\begin{array}{c}\text { Percent of } \\
\text { patients }\end{array}$ \\
\hline Oncological disease & 21 & 38.2 \\
\hline Abdominal cancer & 11 & 20.0 \\
\hline Lung cancer & 7 & 12.7 \\
\hline Gynecological cancer & 6 & 10.9 \\
\hline Haematological cancer & 3 & 5.5 \\
\hline Breast cancer & 3 & 5.5 \\
\hline Prostate cancer & 3 & 5.5 \\
\hline Urinary system cancer & 1 & 1.8 \\
\hline Unknown primary tumor & 55 & 100.0 \\
\hline Number of patients: & & \\
\hline Non oncological disease & 13 & 24.5 \\
\hline Iron deficiency & 13 & 24.5 \\
\hline Unexplained anemia & 7 & 13.2 \\
\hline Angiodysplasia & 7 & 13.2 \\
\hline Rheumatological disease & 3 & 5.7 \\
\hline Abdominal disease & 3 & 5.7 \\
\hline Nephrological disease & 2 & 3.8 \\
\hline B-12 deficiency & 2 & 3.8 \\
\hline Haematological disease & 2 & 3.8 \\
\hline Hematoma & $\mathbf{1 0 8}$ & 1.9 \\
\hline Epistaxis & & 100.0 \\
\hline Number of patients: & & \\
\hline Total number: & & \\
\hline
\end{tabular}

\section{Discussion}

Although blood transfusions are believed to be beneficial for anaemic patients, this hypothesis has never been proven in a prospective controlled clinical trial. The relative benefit-risk ratio of a blood transfusion is an important aspect to inform patients about [6].

The latest Cochrane review found that restrictive transfusion strategies are associated with a statistically significant reduction in hospital mortality, but not of 30-day mortality [7].

The existing evidence supports the use of restrictive transfusion triggers for most patients. It is concluded that, for most patients, giving less blood is safe, and blood transfusions are probably not essential until haemoglobin levels drop below 4.3 to $5.0 \mathrm{mMol} / \mathrm{L}$, so long as there is no notable bleeding or known coronary artery disease. The benefits of minimising allogeneic red blood cell transfusion are likely to be most pronounced in locations where there is doubt about the safety of the blood supply [7].
Furthermore non-bleeding patients should be transfused with one dose of a blood component at a time, and then be reassessed clinically and by laboratory testing before further transfusions. Consequently, a RBCT of two units is no longer considered as the "standard" dose in non-bleeding patients [8].

Causes of anaemia in patients with cancer are often multifactorial. Anaemia may be attributed to underlying comorbidities, such as bleeding, haemolysis, hereditary disease, renal insufficiency, nutritional deficiencies, anaemia of chronic disease, or a combination of the above. Malignant disease can lead to or exacerbate anaemia when cancer cells directly suppress haematopoiesis through bone marrow infiltration or produce cytokines that lead to iron sequestration. Chronic blood loss at tumour sites and organ damage, the myelo-suppressive effect of chemotherapy and the hematologic toxicity of radiation therapy are other well-known factors leading to anaemia [9].

Despite the fact that multiple studies have shown that RBCT is associated with higher mortality, it is the standard treatment for cancer related anaemia in Denmark. Denmark is provided with an extensive availability of donated blood and easy access to inexpensive blood products because of anonymous, voluntary and unpaid blood donations. In Denmark (population: 5 million) around 278000 portions of red blood cells are transfused each year (2012) [5]. Earlier studies have shown that up to $40 \%$ of cancer patients with chemotherapy-associated anaemia in Western Denmark received a RBCT in an inpatient setting. Patients having solid tumours had a higher rate of anaemia in response to chemotherapy [10].

In our department, patients having abdominal and pulmonary cancer received most of the transfusions among the oncological patients (Table 1).

The National Board of Health in Denmark recommends blood transfusion when $\mathrm{Hb}$ level is below $4.5 \mathrm{mMol} / \mathrm{L}$, and no transfusion over $6.0 \mathrm{mMol} / \mathrm{L}$. Between $4.5-6.0 \mathrm{mMol} / \mathrm{L}$ it is the physician's motivated decision that determines whether or not a patient receives a transfusion. The purpose of transfusing anaemic patients with malignant disease is to overcome the anaemic symptoms such as fatigue and dyspnea [11]. As expected; the referral pattern confirmed that there was a cooperation pattern among the general practitioners and the oncological departments. Together they referred $89 \%$ of all the anaemic patients.

Patients with chemotherapy induced anaemia experience an important time burden when being treated with RBCT in addition to the time burden already added by chemotherapy [2]. This current way of expeditious transfusions may increase these patients' quality of life because they can avoid longer hospitalization.

The group of unexplained anaemia was included in the nonmalignant group, until the opposite was established. This might be information bias, and some of the patients might become included in the oncological group in a follow up study.

We have not had any serious complications like transfusion 


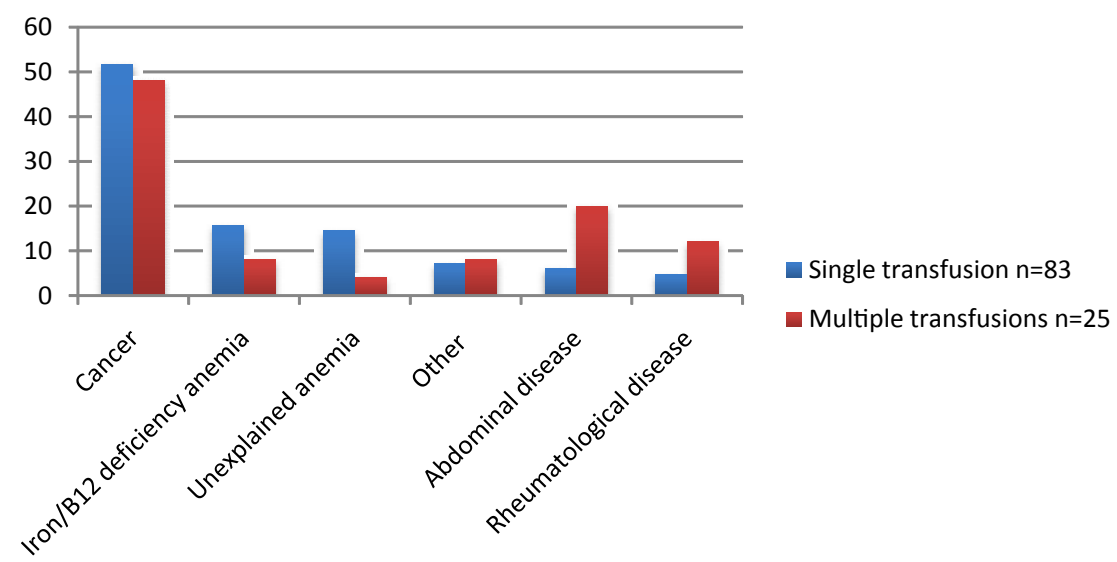

Figure 1: Patients receiving single and multiple transfusions in percent grouped after disease.

related acute lung injury. There was no long term follow up, which would have given a greater total perspective of the pros and cons of expeditious blood transfusions. We have noted that 11 patients died during the review period, 7 of them had a diagnosed cancer.

This is a practical example of giving anaemic patients RBCT in an uncomplicated and expeditious way close to home and followed by continued treatment and investigation at hospital or with their general practitioner.

\section{Conclusion}

Non-trauma related red blood cell transfusions may be given expeditiously in a QDU integrated in an ED setting with very limited waiting time. On average oncological patients received RBCT at a higher Hb level than other anaemic patients. The transfusion trigger for patients with non-oncological disease appears to comply with national guidelines [11].

Future possibilities for assessment of the QDU integrated in an ED setting could include other short-time therapies or causes for observation, such as patients with chest pain and a normal ECG, patients admitted for IV antibiotic treatment, and patients referred for assessment of deep venous thrombosis.

\section{References}

1. Petersen DB, Schmidt TA. Quick diagnostic unit integrated in an emergency department setting reduces medical admissions - an observational study. Journal of Hospital Administration [Internet]. 2013 Dec 18 [cited 2014 Oct 9];3(3). Available from: http://www. sciedu.ca/journal/index.php/jha/article/view/3204
2. Shreay S, Desrosiers M-P, Corey-Lisle P, Payne K. A retrospective study to evaluate the time burden associated with outpatient red blood transfusions indicated for anemia due to concomitantly administered chemotherapy in cancer patients. Support Care Cancer. 2013 May;21(5):1335-40. DOI: 10.1007/s00520-012-1671-9

3. Madsen KHB, Rasmussen SW, Petersen DB, Schmidt TA, Impact of Quick Diagnosis Unit Integrated in an Emergency Department Setting. January 2013

4. Dansk Transfusionsdatabase ÅRSRAPPORT 2012.

5. Dansk Transfusionsdatabase ÅRSRAPPORT 2013.

6. Goodnough LT, Levy JH, Murphy MF. Concepts of blood transfusion in adults. The Lancet. 25 May 2013, 381 (9880): 1845 - 1854. (http:// press.thelancet.com/blood1.pdf)

7. Carless PA, Henry DA, Carson JL, Hebert PP, McClelland B, Ker K. Transfusion thresholds and other strategies for guiding allogeneic red blood cell transfusion. Cochrane Database Syst Rev. 2010;(10):CD002042.

8. Murphy MF, Waters JH, Wood EM, Yazer MH. Transfusing blood safely and appropriately. BMJ. 2013;347:f4303.

9. Rodgers GM, Becker PS, Blinder M, Cella D, Chanan-Khan A, Cleeland $C$, et al. Cancer- and chemotherapy-induced anemia. J Natl Compr Canc Netw. 2012 May;10(5):628-53.

10. Yong M, Riis AH, Fryzek JP, Møller BK, Johnsen SP. Predictors and patterns of red blood cell transfusion use among newly diagnosed cancer patients with chemotherapy-associated anemia in Western Denmark (1998-2003). Clin Epidemiol. 2011;3:91-9.

11.Sundhedsstyrelsen. Vejledning om Blodtransfusion. København: Sundhedsstyrelsen. 